\title{
Die Didaktisierung von Phraseologismen im DaF-Unterricht anhand multimodaler Texte
}

\author{
Nadine Rentel (Wien)
}

\begin{abstract}
Semantically complex units cover a high percentage of the lexicon of a language, ranging from collocations or routine formula to proverbs. The difficulty for learners of a foreign language lies in the fact that in many cases the meaning of those elements of the lexicon does not result just from addition of the meaning of individual constituents. Although (active and passive) knowledge about such lexical elements can be considered essential for successful communication in a foreign language, there still exists a lack (we focus on German as a foreign language, but we assume that the situation is comparable for other languages) of upto-date and authentic didactic material that takes into account the characteristics of semantically complex units and the learners' cognitive strategies to analyze their meaning. In this paper we develop a didactic model that describes the use of magazine advertisements in teaching German as a foreign language. Due to their multimodality (e.g. the combination of verbal and visual elements) the texts allow the learners to better understand and actively use semantically complex units, even with a low competence level. In order to illustrate our didactic approach we present 5 advertisements that we have used with learners of German in their first and second year at a French university. It turns out that not only can phraseology be conveyed in an efficient way but that other educational objectives, such as a general increase of the learners' motivation, can be achieved.
\end{abstract}

\section{Einleitung und theoretische Vorüberlegungen}

Phraseologismen als feste syntaktische und semantische Einheiten stellen im fremdsprachlichen Deutschunterricht unter anderem deswegen eine besondere Herausforderung dar, weil sich ihre Gesamtbedeutung nicht aus der reinen Addition der Sememe ihrer einzelnen Bestandteile ergibt ${ }^{1}$. Hallsteinsdóttir und Farø argumentieren, dass gerade "[d]ie Lexikalisierung als verbale Zeichen mit einer konventionell festgelegten, einheitlichen Bedeutung [...] bei den Phraseologismen dazu [führt], dass die phraseologische Bedeutung nicht der Addition der Bedeutungen der einzelnen Komponenten entsprechen kann" (Hallsteinsdóttir/Farø 2010: 133). Als Begründung führen die Autoren die Tatsache an, dass als Folge der Lexikalisierung "die Komponentenbedeutung prinzipiell sekundarisiert [wird] und die Zeichenbedeutung des Phraseologismus [...] an ihre Stelle [tritt]" (ebd.). Zudem transportieren diese komplexen lexikalischen Einheiten häufig kulturspezifisches Wissen, was ihr Verständnis für Fremdsprachenlerner zusätzlich erschwert. Neben dem

1 Zur Kategorisierung von Phraseologismen und zur theoretischen Reflexion siehe z. B. Burger 1998 und Lüger 1997. Im vorliegenden Beitrag sollen die wissenschaftlichen Vorüberlegungen in den Hintergrund treten und stattdessen der Fokus auf die Phraseodidaktik als einem Teilbereich der Phraseologieforschung gelegt werden (vgl. die ausführliche Darstellung zur Phraseodidaktik des Deutschen bei Hallsteinsdóttir in der Einleitung dieses Themenhefts). 
Problem der korrekten Interpretation phraseologischer Einheiten besteht überdies, abhängig von vielfältigen Kriterien wie der Textsorte, dem Grad an Idiomatizität und der Gebrauchsfrequenz phraseologischer Ausdrücke sowie dem Kompetenzniveau der Lerner die Schwierigkeit, diese im Text zu identifizieren (cf. Anisimova 2002: 245).

Phraseologismen nehmen jedoch einen erheblichen Anteil des deutschen Wortschatzes ein, und das aktive und passive Beherrschen zumindest eines Teils dieser lexikalisch komplexen Einheiten ist für künftige Germanisten, Deutschlehrer, aber auch Übersetzer oder Mitarbeiter in deutschsprachigen Unternehmen unabdingbar (cf. ebd.). Die Auffassungen zur Rolle der Phraseologie im Fremdsprachenunterricht unterliegen einem diachronen Wandel (vgl. dazu ausführlich die unterschiedlichen Phasen der Phraseodidaktik bei Hallsteinsdóttir in der Einleitung dieses Themenhefts). Mittlerweile scheint in der Forschung jedoch weitgehend Einigkeit darüber $\mathrm{zu}$ bestehen, dass "Phraseologismen für die fremdsprachliche Kommunikation unverzichtbar sind" (Hallsteinsdóttir, in der Einleitung des Themenhefts) und dass aus diesem Grund der Phraseodidaktik, d.h. "der systematischen Vermittlung von Phraseologismen" (Ettinger 2001: 87) im fremdsprachlichen Deutschunterricht besondere Aufmerksamkeit gewidmet werden sollte²:

$\mathrm{Da}$ der Phraseologie für den Fremdsprachenunterricht eine besondere Bedeutung zukommt, dürfte außer Frage stehen. Die Befähigung zu einer auch nur begrenzten Kommunikation in einer Fremdsprache ist ohne eine minimale Beherrschung der Phraseologie nicht möglich. (Fleischer 1982: 32)

Werbekommunikate allgemein und Werbeanzeigen im Besonderen eignen sich aus verschiedenen Gründen für einen phraseodidaktischen Einsatz im fremdsprachlichen Unterricht. Ein Vorteil im Vergleich zu anderen Textsorten ist der zentrale Stellenwert von Werbeanzeigen in unserer Alltagskultur (cf. Stöckl 2004: 233), wodurch die Studierenden bereits Erfahrungen mit dem Unterrichtsgegenstand gesammelt haben und daher in der Lage sind, zum Unterrichtsgespräch beizutragen. Ein weiterer Vorteil ist die relative Kürze und Überschaubarkeit der Texte, was den Zugang für die Kursteilnehmer bereits auf niedrigen Kompetenzniveaus erleichtert. In der werblichen Kommunikation werden weiterhin kulturelle Werte einer Sprachgemeinschaft vermittelt (zur Kulturgebundenheit von Werbung cf. Hahn 2000: 28ff.; Schroeder 1994: 33; Stöckl 2004: 244), so dass neben fremdsprachlichen Fertigkeiten das landeskundliche Wissen erweitert werden kann. In Werbeanzeigen wird zudem nicht nur das Standarddeutsche verwendet, sondern regionale oder jugendsprachliche Varietäten sowie Anglizismen werden mit dem Ziel eingesetzt, die Aufmerksamkeit der Rezipienten zu erregen und die Werbebotschaft möglichst dauerhaft in deren Gedächtnis zu verankern. Durch den Einsatz von Werbeanzeigen im FSU ist es weiterhin möglich, unterschiedliche Fertigkeiten zu trainieren, etwa das Leseverstehen, die eigene Textredaktion und Strategien der mündlichen Präsentation.

Das zentrale Argument für den Einsatz von Werbeanzeigen in der Phraseodidaktik ist jedoch in der semiotischen Komplexität der Texte begründet. Aufgrund ihrer Multimodalität (dies meint die synergetische Verknüpfung von Sprache und Bild auf der formalen und inhaltlichen Ebene) werden in Werbeanzeigen in vielen Fällen mehrere der potenziellen Lesarten von Phraseologismen, mindestens die kompositionelle (also wörtliche) und die phraseologische, aktiviert (vgl. Hallsteinsdóttir/Farø 2010: 133)³. Für die Didaktisierung von Phraseologismen

2 Mehrere Autorinnen und Autoren vertreten die Auffassung, dass eine minimale Kenntnis des phraseologischen Wortschatzes, insbesondere der Phraseologie im weiteren Sinne, eine notwendige Voraussetzung für das erfolgreiche Kommunizieren in der Fremdsprache darstellt, was wiederum ein Argument für den möglichst frühen Einsatz der Phraseodidaktik liefert (vgl. z. B. Aguado 2002: 43, Daniels/Pommerin 1979: 575 und Jesenšek 2006: 138).

3 Zur Multimodalität von Werbeanzeigen und zu den vielfältigen Verknüpfungsstrategien von Sprache und Bild auf der formalen und inhaltlichen Ebene vgl. Rentel 2005. Die Aktivierung der kompositionellen (= 
im FSU bietet diese visuell-verbale Doppelkodierung den Vorteil, dass sich sowohl die Transparenz (und damit die Verständlichkeit) als auch die Merkbarkeit erhöhen. Ein weiteres Argument für den hier vertretenen phraseodidaktischen Ansatz besteht in der bereits weiter oben angeführten Auffassung, dass sich die Bedeutung von Phraseologismen eben nicht durch die Kenntnis ihrer (verbalen) Komponentenbedeutungen erschließen lässt. Mit Hallsteinsdóttir/Farø (2010: 134) argumentieren wir, dass insbesondere "[e]in Nichtmuttersprachler, der den Phraseologismus nicht kennt, [...] die phraseologische Bedeutung häufig nicht richtig [wird] rekonstruieren können", wenn er lediglich auf die sprachliche Form zurückgreift. Hier bieten semiotisch komplexe Werbeanzeigen die Möglichkeit, Phraseologismen durch das Zusammenspiel bildlicher und sprachlicher Informationen transparent und analysierbar zu machen. Durch die Segmentierung der komplexen Sequenzen und die damit verbundene kompositionelle Verarbeitung werden Zusammenhänge zwischen den einzelnen Bestandteilen des Phraseologismus erkennbar, so dass diese Komponenten nach einer Phase der Festigung produktiv in anderen kommunikativen Kontexten verwendet werden können (vgl. Aguado 2001: 40 und Hallsteinsdóttir 2001: 303f.).

Ziel des Beitrags ist es einerseits, zu diskutieren, inwieweit Werbeanzeigen es ermöglichen, gezielt den phraseologischen Wortschatz des Deutschen im FSU zu erarbeiten und andererseits durch eine kurze Darstellung der eigenen Lehrerfahrung in diesem Bereich zu zeigen, wie durch den Einsatz dieser Textsorte weitere Lernziele erreicht werden können. Weiterhin soll dem konkreten Desiderat nach dem Erstellen aktueller phraseodidaktischer Übungsmaterielien (vgl. Hallsteinsdóttir in der Einleitung dieses Themenhefts) Rechnung getragen werden. Die im Folgenden diskutierten, ausgewählten Beispiele aus der deutschen Anzeigenwerbung sind unterschiedlichen Ausgaben des Spiegel aus dem Jahr 2009 entnommen.

\section{Das phraseodidaktische Modell}

Das im Folgenden dargestellte Modell der Didaktisierung von Phraseologismen im universitären DaF-Unterricht anhand von Werbeanzeigen wurde mit französischen Studierenden erprobt. Es handelte sich um Studierende der Germanistik und der Angewandten Fremdsprachen aus dem ersten und zweiten Lernjahr, die bei Eintritt in die Universität in der Regel ca. 8 Jahre Deutsch an der Schule gelernt haben. Trotz dieser formalen Qualifikationsvoraussetzung sieht sich der Fremdsprachenlehrer an der Universität bezüglich des sprachlichen Kompetenzniveaus einer relativ heterogenen Zielgruppe gegenüber. Dies erfordert eine sprachniveaudifferenzierte Übungstypologie, die darin bestehen kann, zu Beginn verstärkt die passive Ebene zu trainieren (z. B. durch Übungen zum Identifizieren und Verstehen sehr frequenter Routineformeln aus der Alltagskommunikation) und mit zunehmendem Kompetenzniveau die aktiven Anteile zu erhöhen. Letzteres könnte beispielsweise im Erstellen eigener Werbekommunikate münden, die Phraseologismen enthalten. Die nachstehend vorgestellte Übungstypologie ist daher nur ein Vorschlag zur Unterrichtsgestaltung, wobei die einzelnen Arbeitsaufträge an das jeweilige Sprachniveau der Lernergruppe angepasst bzw. den einzelnen Übungsblöcken ein unterschiedlich hoher Stellenwert zugewiesen werden sollte. Methodisch lehnt sich das didaktische Modell an den phraseologischen Dreischritt nach Kühn $(1992,1994)$ an.

Im Unterricht sollen die Studierenden anhand ausgewählter deutscher Belege zunächst die Werbeanzeige beschreiben, wobei sie sowohl das vorher eingeführte Fachvokabular zur

wörtlichen) Bedeutung von Phraseologismen durch die Verankerung verbaler Schlüsselelemente im bildlichen Anzeigenteil wird als Remotivierung bezeichnet. Die phraseologische Bedeutung bleibt dabei erhalten. 
Identifizierung der Anzeigenbestandteile als auch den Wortschatz zur Bildbeschreibung verwenden sollen. In einem zweiten Schritt sollen sie den Phraseologismus, der sich in der Regel in der Headline befindet, identifizieren und benennen, bevor eine Erläuterung des Bezugs des Phraseologismus zum visuellen Anzeigentext erfolgt. Die Überprüfung, ob die Bedeutung des Phraseologismus erfasst wurde, ist durch deren Paraphrasierung möglich. Im Anschluss daran werden die remotivierte (wörtliche) und die phraseologische Bedeutung kritisch gegenübergestellt, die Relevanz beider Lesarten für die Werbebotschaft aufgezeigt und diskutiert, in welcher Weise sich die zentrale Werbebotschaft aus der Sprache-BildKombination konstituiert. Im Rahmen des interkulturellen Lernens sollen die Teilnehmer weiter überlegen, ob es eine vergleichbare phraseologische Einheit in ihrer Muttersprache gibt und worin Unterschiede und Gemeinsamkeiten zwischen dem Französischen und dem Deutschen bestehen. Als Kommunikationsübung zur freien mündlichen Ausdrucksfähigkeit sollen die Studierenden dann ihre eigene Meinung zu Kriterien wie Ästhetik, Inhalt oder Aufbau der Werbeanzeige äußern und Hypothesen zu ihrer Wirksamkeit anstellen.

Schematisch stellt sich der Arbeitsauftrag wie folgt dar:

1) Mündliche Beschreibung der Werbeanzeige und ihrer Bestandteile.

2) Identifizieren des Phraseologismus. [= ERKENNEN]

3) Erläutern (= Paraphrasieren) des Phraseologismus und des Bezugs zum bildlichen Anzeigenteil. [= ENTSCHLÜSSELN]

4) Gegenüberstellen der wörtlichen und der phraseologischen Bedeutung.

5) Inbezugsetzen der semiotischen Anzeigengestaltung zur Werbebotschaft.

6) Kontrastierung mit der Muttersprache (hier: Französisch).

7) Eigene Stellungnahme.

Den konkreten Einzelanalysen von Werbeanzeigen schließt sich im Unterricht eine Phase der Festigung an, in deren Rahmen das aktive und passive Beherrschen von Phraseologismen anhand unterschiedlicher Übungstypen systematisch trainiert wird [= ERWERBEN]. Als besonders effizient hat sich hierbei das Bilden eigener Sätze erwiesen, die die im Unterricht zuvor besprochenen Phraseologismen enthalten; zu möglichen Übungsformen wie zum Beispiel Substitutions- oder Ergänzungsübungen cf. Anisimova 2002: 251ff.; Ettinger 2007; Lüger 1997. Am Ende der Unterrichtseinheit sollen die Kursteilnehmer in Kleingruppen eigene multimodale Werbeanzeigen entwickeln, die Phraseologismen enthalten und diese im Plenum vorstellen. Auf diese Weise werden neben der mündlichen Ausdrucksfähigkeit allgemeine Präsentationstechniken eingeübt.

\section{$3 \quad$ Materialien und Übungsformen}

Im Folgenden sollen anhand von 5 Anzeigenbeispielen und jeweils eines Erklärungsansatzes die Möglichkeiten der Didaktisierung multimodaler Werbeanzeigen, die Phraseologismen enthalten, im DaF-Unterricht illustriert und somit Anregungen für eigene Vermittlungsansätze gegeben werden. Die Auswahl der hier dargestellten Werbeanzeigen beruht auf der Berücksichtigung unterschiedlicher Produktkategorien und möglichst vielfältiger Strategien der formalen Verknüpfung bildlicher und sprachlicher Elemente. Einschränkend muss hinzugefügt werden, dass die Arbeit mit Phraseologismen in Werbeanzeigen zu einem gewissen Anteil immer vom zur Verfügung stehenden Materialkorpus abhängt. Ein systematisches Erarbeiten ausgewählter Inhaltsbereiche kann daher nicht immer gewährleistet werden. Diese Einschränkung wird m. E. jedoch durch die bereits dargelegten Vorteile des Einsatzes der Textsorte in der Phraseodidaktik sowie durch die Tatsache, dass die darin enthaltenen Phraseologismen sich durch einen hohen Grad an Aktualität und eine hohe 
Gebrauchsfrequenz ${ }^{4}$ auszeichnen, aufgewogen. Sämtliche Beispielanalysen wurden im eigenen universitären Unterricht eingesetzt.

\subsection{Analysebeispiel I: Saab 93}

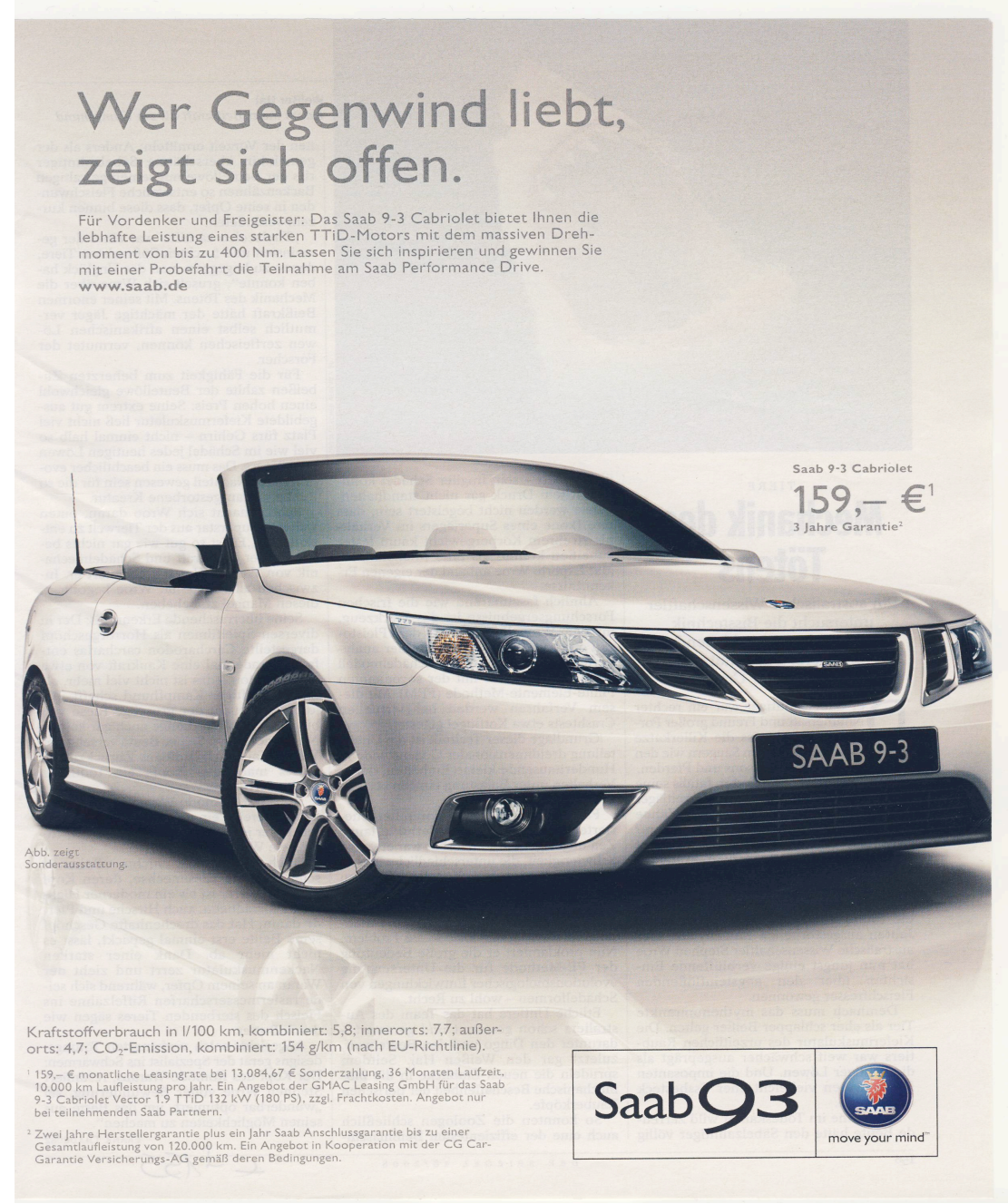

Abb. 1: Analysebeispiel I (Der Spiegel 10/2009: 26)

Wie in sämtlichen im Unterricht eingesetzten Beispielen befindet sich der Phraseologismus in der Headline, nicht etwa im Slogan (Move your mind.) ${ }^{5}$, da letzterer in mehreren Werbeanzeigen, manchmal auch für eine ganze Kampagne, identisch ist und ein Wiedererkennen einer Marke gewährleisten soll. Die Headline hingegen ist in jeder Anzeige formal und inhaltlich anders gestaltet, wodurch zielgerichtete und kreative Sprache-BildBezüge und damit ein Remotivieren phraseologischer Einheiten erst möglich werden.

4 Die Kriterien der Aktualität und der Frequenz der verwendeten sprachlichen Elemente können aufgrund der kommunikativen Ziele (Ansprache einer möglichst hohen Zahl an Rezipienten, Abheben von Konkurrenzbotschaften durch einen kreativen Sprachgebrauch) und Kommunikationsbedingungen von Werbung vorausgesetzt werden.

5 Gründe für das vermehrte Auftreten von Phraseologismen in diesem Anzeigenteil liegen in der funktionalen Affinität von Headline und visuellem Anzeigentext: Beide sollen die Aufmerksamkeit des Rezipienten erregen, sie kommunizieren die grundlegende Werbebotschaft und die Sprache-Bild-Bezüge sind zwischen Headline und bildlichem Anzeigenteil am deutlichsten ausgeprägt (cf. Rentel 2005). 
Im vorliegenden Beispiel wäre die Interpretation der Headline Wer Gegenwind liebt, zeigt sich offen ohne die Rezeption des visuellen Teiltextes vermutlich abstrakter Art (dies betrifft die zentralen lexikalischen Einheiten Gegenwind sowie sich offen zeigen): 'wer Diskussionen/Auseinandersetzungen/Kontroversen liebt, der ist Gegenargumenten und alternativen Sichtweisen gegenüber aufgeschlossen'. Durch das abgebildete Automobil (Cabrio) erfolgt eine Remotivierung der Headline, die nun wörtlich genommen und in einer zweiten, konkreten Lesart verstanden wird: 'wer den bei der schnellen Fahrt im Cabrio entstehenden Gegenwind/Fahrtwind liebt, der fährt offen (= Cabrio mit geöffnetem Verdeck fahren)'. Das grundlegende Prinzip der visuell-verbalen Remotivierung von Phraseologismen, welches im Wörtlichnehmen bestimmter lexikalischer Bestandteile durch einen Referenzbezug auf visuelle Schlüsselelemente im visuellen Anzeigenteil besteht, wird deutlich und wurde von den Studierenden auch so erkannt, ebenso wie die Tatsache, dass nicht etwa die kompositionelle Bedeutung die phraseologische verdrängt, sondern dass beide Lesarten in gleichem Maße relevant sind für die Kommunikation der Werbebotschaft. Im vorliegenden Beispiel haben die Lernenden die wörtliche Bedeutung der Ebene der konkreten Produktvorteile (das Fahren mit geöffnetem Verdeck) zugewiesen, die phraseologische Bedeutung wurde hingegen auf das übergeordnete Markenimage (Weltoffenheit, Toleranz und Neugier) bezogen.

\subsection{Analysebeispiel II: $3 \mathrm{M}$}

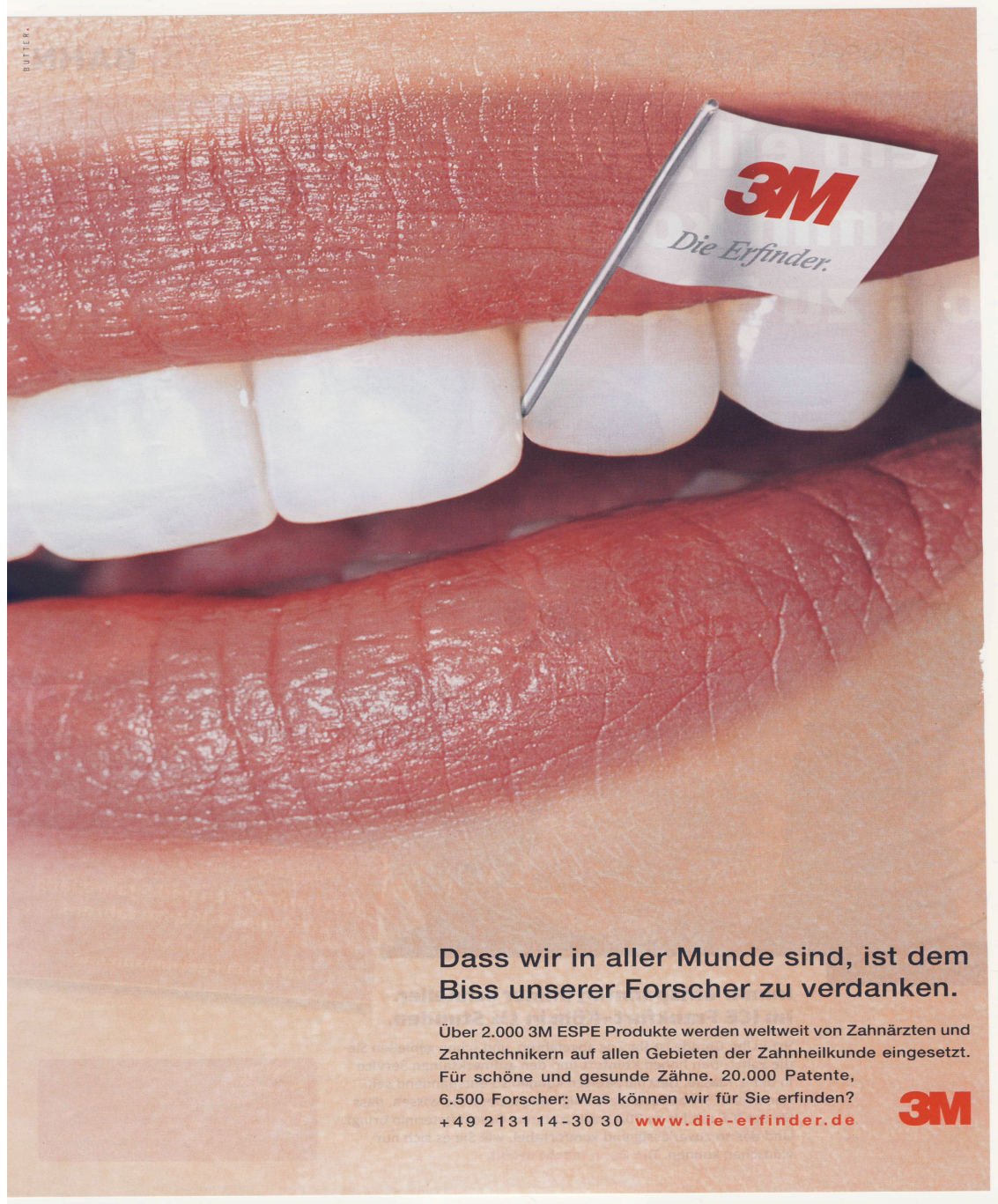

Abb. 2: Analysebeispiel II (Der Spiegel 13/2009: 45) 
In diesem Beispiel werden gleich zwei Phraseologismen in der Headline durch den visuellen Anzeigenteil remotiviert. Dieser Beleg zeichnet sich daher durch einen besonders hohen didaktischen Nutzen für den DaF-Unterricht aus. Im ersten Teil der Headline geht es um die feststehende Wortverbindung in aller Munde sein: 'sehr bekannt sein, aktuelles Thema sein', während im zweiten Teil der Phraseologismus Biss haben: 'Durchsetzungsvermögen haben' im Zentrum steht (die Bedeutungsparaphrasen basieren auf dem Duden).

Bei einer alleinigen Dekodierung der Headline als verbalem Anzeigenbestandteil (Dass wir in aller Munde sind, ist dem Biss unserer Forscher zu verdanken) dominiert die phraseologische Bedeutung: 'dass alle so positiv von uns reden, verdanken wir dem Ehrgeiz unserer Forscher' (dies entspricht der zentralen Werbeaussage). Die Aufmerksamkeit der Rezipienten wird nun durch die Großaufnahme eines leicht geöffneten weiblichen Mundes, zwischen dessen Schneidezähnen ein kleines Fähnchen mit der Aufschrift des Herstellers steckt, erregt, wodurch die Remotivierung erfolgt, d. h. das wörtliche, bildhafte Interpretieren der verbalen Aussage, basierend auf der Auflösung der Phraseologismen in ihre Einzelbestandteile und der damit einhergehenden Relativierung ihrer Idiomatizität. Anders als im ersten Beispiel haben die Studierenden der jeweils phraseologischen Bedeutung der beiden Headline-Bestandteile eine größere Relevanz zugewiesen als der kompositionellen, jedoch haben Umfragen im Kurs ergeben, dass aufgrund des eingängigen Sprache-Bild-Bezugs die phraseologische und für die Werbebotschaft zentrale Aussage besonders gut erinnert wird.

\subsection{Analysebeispiel III: BP}
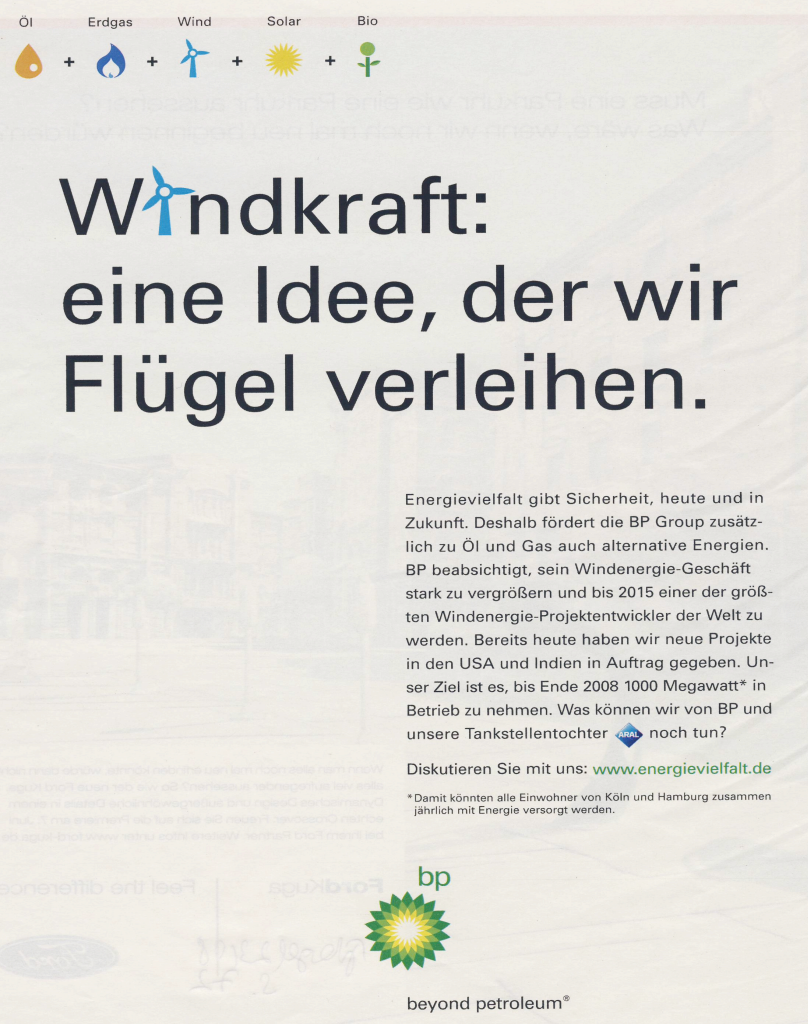

Abb. 3: Analysebeispiel III (Der Spiegel 13/2009: 87)

Im vorliegenden Beispiel werden Prinzipien der inhaltlichen und formalen Verknüpfung von Teiltexten miteinander vereinbart, was die Motivation bei der Dekodierung durch die Fremdsprachenlerner zusätzlich steigern kann: Auf der formalen Ebene fällt auf, dass 
innerhalb des Lexems Windkraft das Graphem I durch die Abbildung einer Windkraftanlage substituiert worden ist und sich dieses Headline-Element somit aus verbalen und visuellen Teilen zusammensetzt. Das Substantiv weist dadurch neben seinem rein symbolischen Charakter auch ikonische Merkmale auf. Es handelt sich um eine Ähnlichkeitsbeziehung (zur triadischen Klassifizierung semiotischer Zeichen vgl. Peirce, sehr übersichtlich und detailliert dargestellt in Keller 1995: 160ff.) zwischen dem visuellen und dem verbalen Element, genauer gesagt um eine visuell-verbale Analogie (zu einer Kategorisierung visuell-verbaler Verknüpfungsstrategien cf. Rentel 2005): Die Windkraftanlage verweist auf das abstrakte Prinzip der Windkraft.

Im zweiten Teil der Headline wird der Phraseologismus jmd./etwas Flügel verleihen ('jmd. anspornen, beflügeln') verwendet, der nun einerseits innerhalb des verbalen Textes durch das Lexem Windkraft remotiviert wird, andererseits durch die Abbildung der Windkraftanlage mit ihren Flügeln. So ergeben sich zwei parallele Interpretationen: 'die alternativen Energien in Form von Windkraft anhand strategischer Konzepte fördern' einerseits und andererseits ganz konkret 'die Windkraftanlagen technisch ausstatten'.

In diesem Beleg erweist es sich wiederum als Vorteil, dass beide Lesarten (sowohl die kompositionelle als auch die phraseologische) des Phraseologismus für die Werbebotschaft relevant sind. Diese doppelte Verankerung erweist sich als positiv sowohl für das Verständnis als auch für die Behaltensleistung und macht deutlich, dass gerade in Werbeanzeigen die potenziellen Lesarten in keiner Weise völlig zusammenhanglos nebeneinanderstehen, sondern in vielen Fällen gezielt unterschiedliche Aspekte des beworbenen Produkts hervorheben. Durch dieses Oszillieren ist es möglich, die Lernenden für das Spannungsfeld von wörtlicher und phraseologischer Bedeutung zu sensibilisieren. Weiterhin gaben die Studierenden an, dass sich die Verständlichkeit des Phraseologismus insbesondere durch die Ikonizität der Headline erhöht hat.

\subsection{Analysebeispiel IV: Pioneer Investments}

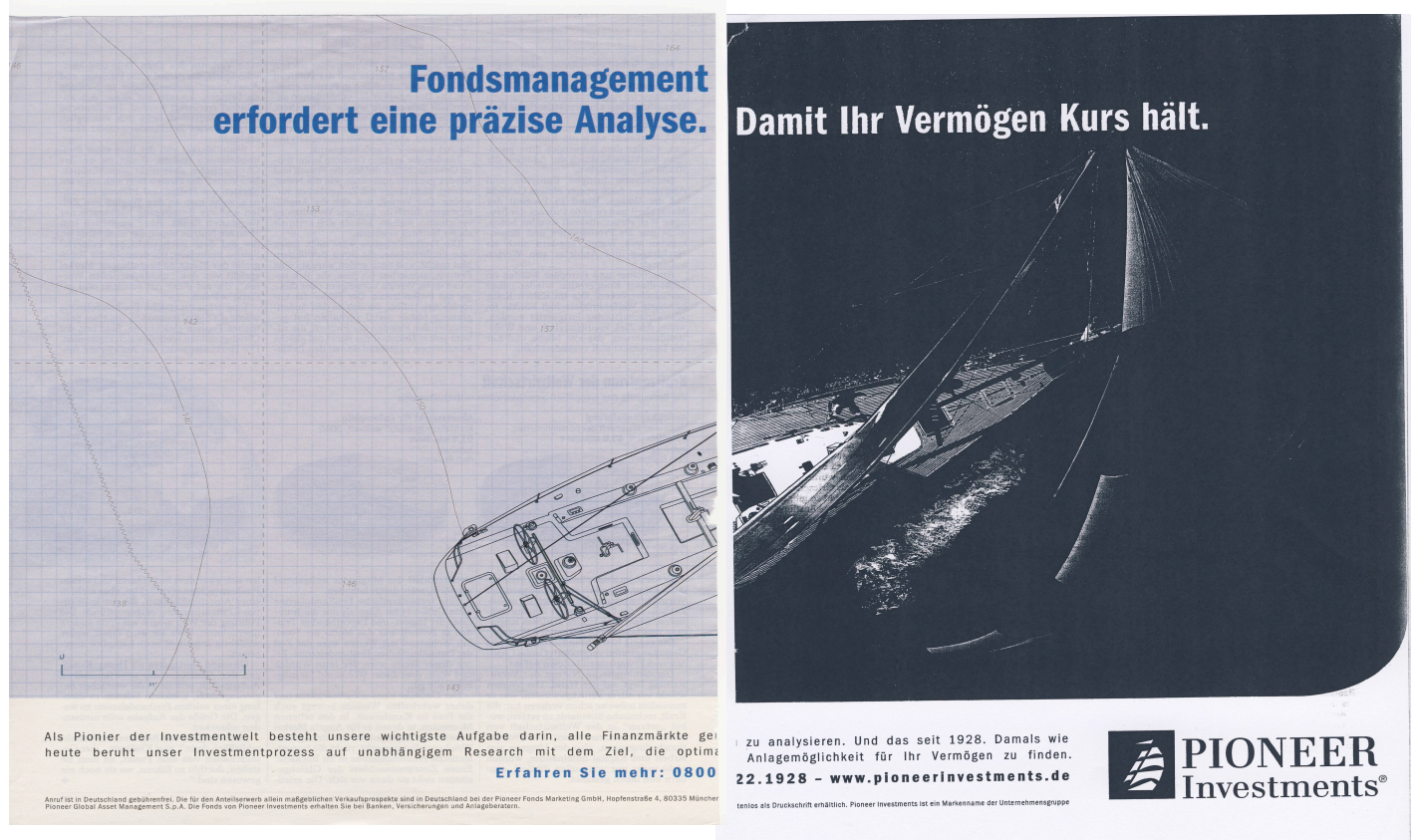

Abb. 4: Analysebeispiel IV (Der Spiegel 7/2009: 43f.) 
Im Rahmen dieser doppelseitigen Werbeanzeige werden gleich zwei zentrale Aussagen des Fondverwaltungsunternehmens verbal und visuell kommuniziert: Einerseits wird eine präzise Analyse der Aktienmärkte herausgestellt, was sich im visuellen Teil der Anzeige durch eine technische Planzeichnug eines Segelbootes äußert. Auf der zweiten Doppelseite der Anzeige wird in der Headline die feststehende Wendung Kurs halten verwendet, die durch den bildlichen Anzeigentext wörtlich genommen wird; das sich vorher noch in der Planung befindliche Segelboot sticht nun in See. Durch gut durchdachte Anlagestrategien des Vermögensmanagements werden keine negativen Kursentwicklungen erwartet, das Vermögen "kommt nicht vom Kurs ab".

\subsection{Analysebeispiel V: ZDF-Magazin Frontal}

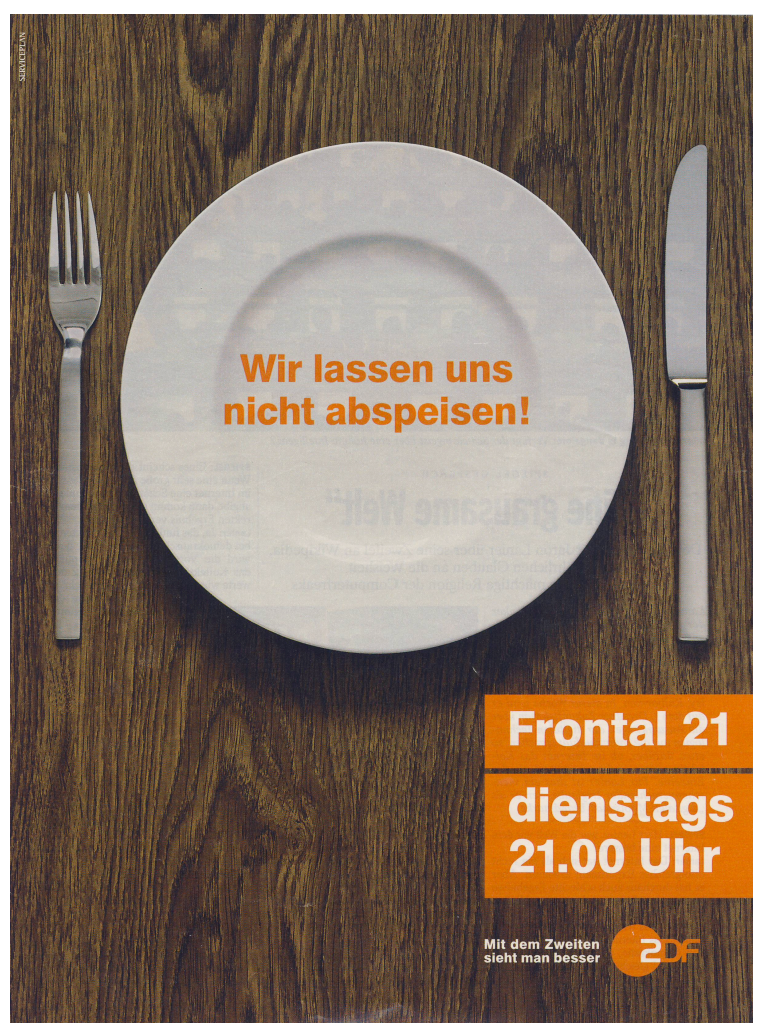

Abb. 5: Analysebeispiel V (Der Spiegel 15/2009: 17)

Die Headline Wir lassen uns nicht abspeisen enthält einen Phraseologismus, dessen Bedeutung paraphrasiert werden kann mit sich (nicht) abspeisen lassen: 'sich (nicht) entmutigen/ohne Erklärung abwimmeln lassen'. Durch den visuellen Anzeigentext erfolgt ein Wörtlichnehmen des Phraseologismus, der durch die Abbildung des leeren Tellers mit den Besteckteilen zu beiden Seiten die konkrete, wörtliche Lesart 'speisen = essen' zugewiesen bekommt. Aufgrund des leeren Tellers wird der Eindruck erweckt, der Gast werde nicht gut bedient und müsse sich mit einem leeren Teller zufrieden geben. Hierdurch wird wiederum die oben genannte abstrakte Lesart evoziert. Im Kontext einer Werbeanzeige für das ZDFPolitmagazin Frontal bezieht sich das gesamte Kommunikat auf den investigativen und kritischen Journalismus, jedoch wird das Personalpronomen in der 1. Person Plural (wir) im visuellen Anzeigentext nicht aufgelöst, sondern erst durch den Slogan und das Logo.

\section{$4 \quad$ Zusammenfassung und Ausblick}

Der Einsatz semiotisch komplexer Werbeanzeigen im DaF-Unterricht an französischen Universitäten hat sich in mehrfacher Hinsicht als didaktisch sinnvoll erwiesen. Die allgemeine Reaktion der Studierenden auf die Textsorte war durchweg positiv, ebenso die erzielten 
Lerneffekte. Konkret bestehen diese in der Erweiterung der Rezeptionskompetenz hinsichtlich einer im Alltag hoch relevanten Textsorte sowie der Kenntnisse bezüglich eines textsortenbedingt aktuellen und sehr häufigen Ausschnitts aus dem phraseologischen Wortschatzes des Deutschen, was wiederum eine verbesserte Kommunikationsfähigkeit erwarten lässt. Im Folgenden soll skizziert werden, inwieweit die Ziele eines handlungsorientierten FSU durch Phraseodidaktik anhand von Werbeanzeigen erreicht wurden. Durch das große Interesse an Werbekommunikation konnte die Motivation deutlich erhöht werden, da die Studierenden einen unmittelbaren Bezug zu ihrer Alltagsrealität, ihren Berufszielen sowie einen nachvollziehbaren Sinn der Thematik erkennen konnten. Als Konsequenz verliefen die Unterrichtsgespräche sehr lebhaft, die Kursteilnehmer hatten sichtlich Freude an der eigenständigen Analyse von Werbeanzeigen sowie an der Präsentation der Arbeitsergebnisse in Kleingruppen. Auch bei der Gestaltung eigener Werbeanzeigen ließ sich ein hohes $\mathrm{Maß}$ an Kreativität und Eigenständigkeit beobachten. Die Arbeit mit Werbeanzeigen eignet sich m. E. somit - nach Adaption an das sprachliche Kompetenzniveau der Zielgruppe - prinzipiell für sämtliche Kurstypen der Sprachpraxis DaF als Methode zur Steigerung der allgemeinen Motivation der Lerner sowie zum gezielten Trainieren bestimmter Fertigkeiten. Insbesondere die Verständlichkeit und Merkbarkeit von Phraseologismen als feststehenden, kulturgebundenen und damit häufig schwer zu erlernenden Bestandteilen des Wortschatzes lässt sich durch die Arbeit mit semiotisch komplexen Werbeanzeigen merklich verbessern. Im Vergleich zum reinen Auswendiglernen syntagmatisch komplexer Einheiten bietet der Ansatz den Vorteil, dass das kognitive System der Lernenden dauerhaft restrukturiert wird.

Trotz dieser zahlreichen positiven Effekte des Einsatzes von Werbeanzeigen für die Phraseodidaktik sind einige Punkte kritisch zu hinterfragen. Beispielsweise kann nicht mit Sicherheit gesagt werden, welche Bedeutung der Phraseologismen durch die Kombination von Sprache und Bild von den Lernenden behalten wird. Durch die Prägnanz des visuellen Anzeigentextes besteht die Gefahr, dass lediglich die remotivierte Lesart memorisiert wird. Es ist denkbar, die Lernenden auf die mnemotechnische Funktion der bildhaften, konkreten Bedeutung hinzuweisen, damit diese bewusst als Lernstrategie, Verständnis- und Merkhilfe genutzt wird. Man kann zudem kritisch anmerken, dass die Aktivierung der kompositionellen Bedeutung in einigen Fällen das Verständnis erschweren kann, insbesondere wenn kein Zusammenhang zwischen der kompositionellen und der phraseologischen Bedeutung besteht. Wie anhand der Einzelanalysen gezeigt werden wird, ist dies jedoch in Werbeanzeigen in den seltensten Fällen so, da beide Bedeutungen relevant für das Herausstellen von Produktvorteilen sind und in einem Sinnzusammenhang stehen. Da Werbebotschaften von möglichst vielen Rezipienten verstanden und behalten werden sollen, wäre es zudem zu riskant, für den Bereich der Remotivierung von Phraseologismen zu komplexe Strategien zu verwenden, denn bekanntlich steht für die Rezeption von Werbeanzeigen, die in Konkurrenz zu anderen Botschaften stehen, sehr wenig Zeit zur Verfügung. Eine weitere "Schwäche" von Werbeanzeigen besteht in dem relativ begrenzten Repertoire des darin enthaltenen (bzw. des im Unterricht aufgrund begrenzter zeitlicher Ressourcen einsetzbaren) phraseologischen Wortschatzes, da, nicht zuletzt aufgrund der Materialbasis, nur ausgewählte Bildspendebereiche vertreten sind. Themenbereiche können oftmals nicht systematisch erfasst werden. Dieser Nachteil wird m. E. jedoch durch die Aktualität und die Frequenz der Phraseologismen (bedingt durch die kommunikativen Rahmenbedingungen und Ziele von Werbung) wieder ausgeglichen. Phraseologismen sollten jedoch nicht alleine anhand von Werbetexten vermittelt werden, sondern die Didaktisierung sollte stets im Kontext eines Methodenmixes und Repertoires unterschiedlicher Textsorten erfolgen.

Das Didaktisierungsprinzip, die Bedeutung von Phraseologismen anhand multimodaler Texte, wie zum Beispiel Werbeanzeigen, zu erarbeiten, lässt sich $\mathrm{m}$.E. auf alle modernen 
Fremdsprachen übertragen, da Phraseologismen in allen Einzelsprachen ein konstituierender Bestandteil des Wortschatzes sind. Interessant und dem Lernziel zuträglich ist es dabei, im FSU einen interkulturellen Vergleich durchzuführen, um den Lernern die Kulturgebundenheit solcher Wortschatzelemente und somit die unterschiedliche Strukturierung des phraseologischen Wortschatzes $\mathrm{zu}$ verdeutlichen. Doch nicht nur auf die Unterschiede zwischen den Einzelsprachen sollte in diesem Kontext verwiesen werden. Nach neueren Studien bestehen zwischen phraseologischen Einheiten in unterschiedlichen Sprachen mehr Gemeinsamkeiten als bisher angenommen (cf. Jesenšek 2006: 139f.). Herausgestellt werden müssen daher ebenfalls Ähnlichkeiten zwischen der Ausgangs- und der Zielsprache, um diese für den Lernprozess in der Fremdsprache nutzbar zu machen:

So werden die muttersprachlichen phraseologischen Kenntnisse auf die fremdsprachliche Phraseologie übertragen und der muttersprachlichen Phraseologie kommt eine entscheidende Rolle beim Verstehen fremdsprachlicher Phraseme zu. [...] Die muttersprachlichen phraseologischen Kenntnisse sollten deshalb intensiver ausgenutzt werden. (Jesenšek 2006: 139)

Für einen effizienten FSU, der die Synergieeffekte des interkulturellen Lernens nutzt, müssen für unterschiedliche Sprachenpaare "sprachvergleichende [...] phraseologische Lernmaterialien entwickelt und erstellt werden" (Jesenšek 2006: 140). Dies stellt bis heute eine Herausforderung für die Lehrbuchverlage im Bereich der Fremdsprachendidaktik dar (cf. Hessky 1992), nicht zuletzt deshalb, weil die Einbeziehung der Muttersprache in DaFLehrwerke, die für ein breites Zielpublikum und somit für Lernende mit unterschiedlichen Muttersprachen konzipiert sind, einen nicht zu unterschätzenden Aufwand darstellt. In Lehrwerken, die sich an Mitglieder einer Sprachgemeinschaft wenden oder im Rahmen der von der Lehrperson erstellten, das Lehrwerk ergänzenden Zusatzmaterialien ist eine kontrastive Herangehensweise durchaus möglich und wünschenswert (vgl. Hallsteinsdóttir in der Einleitung zu diesem Themenheft).

\section{Literatur}

Aguado, Karin (2002): "Formelhafte Sequenzen und ihre Funktionen für den L2-Erwerb". Zeitschrift für Angewandte Linguistik 37: 27-49.

Anisimova, Elena V. (2002): "Phraseologismen im Unterricht Deutsch als Fremdsprache". Germanistisches Jahrbuch der GUS "Das Wort" 2002: 245-256.

Burger, Harald (1998): Phraseologie. Eine Einführung am Beispiel des Deutschen. Berlin: Erich-Schmidt-Verlag.

Daniels, Karlheinz/Pommerin, Gabriele (1979): "Die Rolle sprachlicher Schematismen im Deutschunterricht für ausländische Kinder". Die Neueren Sprachen 78/6: 572-586.

Ettinger, Stefan (2001): "Vom Lehrbuch zum autonomen Lernen. Skizze eines phraseologischen Grundkurses für Französisch". In: Lorenz-Bourjot, Martine/Lüger, Heinz-Helmut (eds.): Phraseologie und Phraseodidaktik. Wien, Edition Praesens: 87-104. (= Beiträge zur Fremdsprachenvermittlung, Sonderheft 4).

Ettinger, Stefan (2007): "Phraseme im Fremdsprachenunterricht". In: Burger, Harald/Dobrovol'skij, Dmitrij/Norrick, Neal R. (eds.): Phraseologie. Phraseology. Ein internationales Handbuch zeitgenössischer Forschung. An International Handbook of Contemporary Research. Berlin/New York, de Gruyter: 893-908. (= Handbücher zur Sprach- und Kommunikationswissenschaft 28.1 \& 28.2)

Fleischer, Wolfgang (1982): Phraseologie der deutschen Gegenwartssprache. Tübingen: Niemeyer.

Hahn, Stephan (2000): Werbediskurs im interkulturellen Kontext. Semiotische Strategien bei der Adaption deutscher und französischer Werbeanzeigen. Wilhelmsfeld: Egert-Verlag. 
Hallsteinsdóttir, Erla (2001): Das Verstehen idiomatischer Phraseologismen in der Fremdsprache Deutsch. PHILOLOGIA - Sprachwissenschaftliche Forschungsergebnisse 49). http://www.verlagdrkovac.de/volltexte/3-8300-0435-4.htm, Stand 16. Januar 2011.

Hallsteinsdóttir, Erla/Farø, Ken (2010): "Interlinguale Phraseologie: Theorie, Praxis und Perspektiven". Yearbook of Phraseology: 125-159.

Hessky, Regina (1992): "Aspekte der Verwendung von Phraseologismen im Unterricht Deutsch als Fremdsprache". Fremdsprachen lehren und lernen 21: 159-168.

Hessky, Regina (1992): "Phraseolexeme als harte Nuß für die zweisprachige Lexikographie". In: Földes, Csaba (ed.): Deutsche Phraseologie in Sprachsystem und Sprachverwendung. Wien, Edition Praesens: 107-124.

Jesenšek, Vida (2006): "Phraseologie und Fremdsprachenlernen. Zur Problematik einer angemessenen phraseodidaktischen Umsetzung". Linguistik Online 27: 137-147. http://www.linguistik-online.com/27_06/jesensek.pdf, Stand 10. Juni 2010.

Keller, Rudi (1995): Zeichentheorie. Tübingen: UTB-Verlag.

Kühn, Peter (1992): "Phraseodidaktik. Entwicklungen, Probleme und Überlegungen für den Muttersprachenunterricht und den Unterricht DaF". Fremdsprachen Lehren und Lernen 21: 169-189.

Kühn, Peter (1994): "Pragmatische Phraseologie: Konsequenzen für die Phraseographie und Phraseodidaktik". In: Sandig, Barbara (ed.): EUROPHRAS 92 Tendenzen der Phraseologieforschung. Bochum, Brockmeyer: 411-428. (= Studien zur Phraseologie und Paromiologie 1).

Lorenz-Bourjot, Martine/Lüger, Heinz-Helmut (2001) (eds.): Phraseologie und Phraseodidaktik. Wien, Edition Praesens: 87-104. (= Beiträge zur Fremdsprachenvermittlung, Sonderheft 4).

Lüger, Heinz-Helmut (1997): "Anregungen zur Phraseodidaktik". Beiträge zur Fremdsprachenvermittlung 32: 69-120.

Rentel, Nadine (2005): Bild und Sprache in der Werbung. Die formale und inhaltliche Konnexion von verbalem und visuellem Teiltext in der französischen Anzeigenwerbung der Gegenwart. Frankfurt/Main u. a.: Peter Lang.

Schroeder, Michael (1994): "Frankreich - Deutschland. Zwei unterschiedliche Auffassungen von Kommunikation". In: Koch, Ursula et al. (eds): Deutsch-französische Medienbilder. München, Verlag Reinhard Fischer: 24-42.

Stöckl, Hartmut (2004): "Werbekommunikation - Linguistische Analyse und Textoptimierung". In: Knapp, Karlfried et al. (eds.): Angewandte Linguistik. Ein Lehrbuch. Stuttgart, UTB: 233-254. 